\title{
FACTORES PARA LA FORMACIÓN EN INVESTIGACIÓN DEL TRABAJO SOCIAL: APORTES DESDE LA SISTEMATIZACIÓN DEL SEMILLERO INTERFERENCIAS, UNIVERSIDAD DEL QUINDÍO, COLOMBIA*
}

\author{
FACTORS FOR TRAINING IN SOCIAL WORK RESEARCH: CONTRIBUTIONS \\ FROM THE SYSTEMATIZATION OF THE RESEARCH HOTBED \\ INTERFERENCIAS, UNIVERSIDAD DEL QUINDÍO, COLOMBIA
}

Ana María Gil-Ríos**

\section{Resumen}

Objetivo. Comprender los factores del proceso de formación en investigación en trabajo social que han contribuido al fortalecimiento de capacidades y actitudes investigativas de estudiantes y docentes del semillero Interferencias, entre el segundo semestre de 2015 y el primer semestre del 2017. Metodología. Sistematización realizada desde un enfoque comprensivo y un proceso de cinco etapas: delimitación de la experiencia, elaboración de un plan de trabajo, reconstrucción histórica y análisis e interpretación crítica, desde la cual se construyen categorías de análisis. Resultados. Se identificaron como principales categorías para la formación en investigación elementos epistemológicos, pedagógicos y metodológicos. Conclusiones. La opción decolonial e intercultural y el construccionismo social como aporte para la construcción de pedagogías y metodologías otras que orienten la formación en investigación.

Palabras clave: formación en investigación, actitudes y capacidades investigativas, trabajo social.

\footnotetext{
* El presente artículo es resultado de una sistematización de experiencias realizada con integrantes de un semillero y colectivo de estudio adscrito al programa de Trabajo Social de la Universidad del Quindío, Colombia. La investigación se realizó partiendo de un proceso de descripción y delimitación de la experiencia, desde el cual se decidió recuperar el período de tiempo comprendido entre el segundo semestre de 2015 y el primero del 2017. Posteriormente, se definió el eje central como el proceso de formación que se ha llevado a cabo en el semillero y al aporte en el fortalecimiento de actitudes y capacidades investigativas de sus integrantes. Con base en este se construyeron preguntas y objetivos -los cuales se exponen más adelante- y se continuó con la elaboración de un plan de sistematización desde el cual se establecieron tiempos, procedimientos de trabajo y fuentes de información. Seguido se realizó una reconstrucción histórica del proceso vivido y, con una línea de tiempo, se logró ordenar la información. Para continuar con la fase de análisis e interpretación, en primer lugar, se realizó una narración descriptiva de la experiencia y posteriormente un análisis crítico de la misma. Finalmente, se identificaron los principales aprendizajes y se construyen conclusiones, recomendaciones y estrategias de socialización.

*** Universidad del Quindío. Armenia, Colombia. E-mail: amgil@uniquindio.edu.co.

D orcid.org/0000-0002-6507-0195. Google Scholar
}

rev. eleuthera. 21, julio-diciembre 2019, 126-151

Recibido: 25 de febrero de 2019. Aprobado: 10 de mayo de 2019. ISSN: 2011-4532 (Impreso) ISSN: 2463-1469 (En línea) DOI: 10.17151/eleu.2019.21.8. 


\begin{abstract}
Objective. To understand the factors of training in the research process in Social Work, which have contributed to the strengthening of investigative capacities and attitudes of students and teachers part of the research hotbed Interferencias, between the second semester 2015 and the first semester 2017. Methodology. Systematization carried out from a comprehensive approach, and a five-stage process: delimitation of experience, design of a work plan, historical reconstruction, and analysis and critical interpretation, from which analysis categories are constructed. Results. Epistemological, pedagogical and methodological elements were identified as the main categories for research training. Conclusions. The decolonial and intercultural option and social constructionism as a contribution to the construction of pedagogies and other methodologies that guide research training.
\end{abstract}

Key words: training in research, attitudes and investigative capacities, social work.

\title{
Introducción
}

La investigación es componente fundamental de la intervención ya que permite realizar una acción fundamentada acorde a las realidades y situaciones que enfrentan los profesionales en su práctica. Es a partir de la investigación que se puede aportar a la construcción de conocimiento situado y se pueden reconocer saberes otros que, aunque no se gestan en la academia, es necesario visibilizarlos y comprenderlos.

Para el trabajo social investigar es necesario ya que permite visibilizar situaciones, reconocer la complejidad de las realidades sociales y ponerlas en la agenda del debate público. De allí su carácter de compromiso con la vida, la justicia y los derechos (Cifuentes, 2013).

Siguiendo con esta línea, problematizar la investigación implica hacer revisión de los currículos en los programas académicos y analizar la propuesta pedagógica que los fundamenta. Además, tener claro cómo se desarrolla el proceso de formación para la investigación y con ello el ambiente de aprendizaje, los principios pedagógicos y los docentes que la orientan, las relaciones construidas entre estudiantes/profesores y los aspectos metodológicos que se privilegian.

Cuestionar la formación en investigación desde estos referentes, según Falla (2012) "permite ir más allá del ejercicio esquemático de la enseñanza-aprendizaje” (p. 16) e implica potenciar actitudes y capacidades para problematizar la realidad desde una perspectiva crítica y reflexiva que motive el cuestionamiento y la pasión por investigar (Cifuentes, 2014). 
Factores para la formación en investigación del trabajo social: aportes desde la sistematización del semillero Interferencias...

Grassi (2011) propone que las actitudes investigativas deben ser inherentes al ejercicio profesional de los trabajadores sociales sin importar su ámbito de desempeño; si bien, no todos tienen que considerarse investigadores, es necesario que en la práctica cotidiana desplieguen con solvencia capacidades para el análisis profundo y contextualizado de la realidad, para cuestionarse, dudar, asombrarse, darle cabida a lo incierto y en coherencia asumir su intervención con rigor y compromiso.

Desde este marco, la formación investigativa es un desafío de las unidades académicas, y la investigación, por tanto, debe considerarse eje transversal de las mallas curriculares. Para Falla (2012) "un eje transversal es interdisciplinario, recorre la totalidad del currículo, y la totalidad las áreas del conocimiento, las disciplinas y los temas (...) tienen un carácter globalizante” (p. 14), lo cual significa que abarcan diferentes asignaturas evidenciando articulación. Ello implica trabajo colaborativo entre los docentes y disposición para acompañar a los estudiantes en el proceso creativo de la investigación.

El acompañamiento en los procesos de formación investigativa implica reconocer los ritmos e intereses de quienes investigan, potenciar la escucha para recuperar, valorar y orientar las propuestas de los estudiantes (Cifuentes, 2013). De allí que el papel desempeñado por el docente en la formación para la investigación sea importante, en tanto su labor debe trascender el énfasis de contenidos - teóricos y metodológicos-para orientarse hacia la problematización, autorreflexión, para asumir una postura frente al conocimiento, una actitud que le permite al sujeto colocarse ante aquello que quiere conocer, con el fin de construir nuevas comprensiones y conceptualizaciones (Zemelman, 2005).

Con el ánimo de aportar al debate acá esbozado, el presente artículo busca compartir el proceso vivido ${ }^{1}$ y los hallazgos de la sistematización de experiencias realizada con un semillero

\footnotetext{
${ }^{1}$ La descripción de la experiencia fue referente clave para rescatar el proceso vivido desde el semillero, el cual ha tenido como eje articulador la discusión del trabajo social en clave decolonial e intercultural. Desde este punto de referencia, se inicia el recorrido por diferentes ciclos de trabajo denominados: conocimiento, exploración conceptual, reflexión y transformación. Son llamados ciclos en tanto no fueron etapas lineales, y respondieron a una dinámica en espiral. Acompañando el recorrido de cada ciclo, se vivieron los encuentros entre universidades, para realizar articulaciones y discusiones teóricas que permitieran consolidar una investigación colectiva. En resumen: Primer ciclo, "conociéndonos": caracterizado por la familiarización, reconocimiento y construcción de intereses de trabajo. Se definió nombre del colectivo e inició un proceso de consolidación de identidad grupal. Hechos particulares vividos en este recorrido, salida académica al Valle del Cocora y encuentros conversacionales frente a temas de interés con relación a lo profesional. Segundo ciclo, "exploración conceptual y vivencial": para discutir lecturas que permitieran problematizar ideas con respecto a la investigación y la práctica del trabajo social. Se buscó construir un espacio para la reflexión, salir de la dinámica tradicional académica, cada uno manifestó sus ideas de forma diversa (poemas, escritos, cartas, dibujos). Como actividades vivenciales, se realiza el mural de la indignación. Tercer ciclo, "reflexionando-nos, transformando-nos": se continúa con lecturas, problematizadas a la luz de experiencias cotidianas, como técnicas desarrolladas, el foto-sentir, dibujo, la escucha, simbología y tierra, tejiendo pensamientos y círculos de palabra. Con respecto al encuentro entre universidades, denominado "tejiendo redes", cada semestre se realizó en una sede diferente (Armenia, Bogotá, Manizales y Medellín) para trabajar con los semilleros que forman parte de la red. Se contó con la presencia de docentes, estudiantes y egresados quienes compartieron sus experiencias de trabajo durante el semestre y construyeron los lineamientos de una investigación macro.
} 
de investigación ${ }^{2}$, perteneciente al programa de Trabajo Social de la Universidad del Quindío, el cual viene trabajando desde $2015^{3}$, en la opción decolonial e intercultural como perspectiva para la intervención e investigación profesional. Desde entonces ha estado conformado por estudiantes y algunos docentes del programa. Uno de los aspectos más significativos del proceso que ha desarrollado el semillero, es la formación en investigación, la cual se ha construido a partir de diferentes estrategias y actividades, que han potenciado entre sus asistentes capacidades y actitudes investigativas.

El grupo forma parte de una red de semilleros y grupos de investigación con universidades como: Caldas, Antioquia y La Salle. De manera semestral, se hacen reuniones para compartir avances y reflexiones con respecto al trabajo social, desde la perspectiva intercultural y decolonial y construir una investigación conjunta en el tema.

Teniendo en cuenta el proceso del grupo, resulta interesante conocer los posibles aportes que desde esta experiencia se puede hacer a la formación en investigación, y a los profesores y estudiantes involucrados en ella. Resulta necesario escribir sobre las prácticas, cuestionarlas y someterlas al debate, con el objetivo de aportar discusiones acerca de la formación de trabajadores sociales. Esta intención fue la que se constituyó en punto de partida para iniciar la recuperación de la experiencia.

La concepción de sistematización que orientó la propuesta, permitió entenderla como posibilidad para recorrer de manera más consciente las experiencias vividas, como oportunidad para aprender, des-aprender y generar mayor porosidad entre la ciencia formal y otras formas de producción de conocimientos, de saberes que tienen un valor contextual y de acción (Mosquera, 2005).

El eje central fue el "proceso de formación” llevado a cabo en el grupo, para aportar al fortalecimiento de actitudes y capacidades investigativas, para aprender a investigar investigando, de aquí que la pregunta inicial fuera: ¿qué factores del proceso de formación en investigación, han contribuido al fortalecimiento de capacidades y actitudes investigativas de

\footnotetext{
2 "Interferencias" es el nombre del semillero de investigación, escogido colectivamente por sus estudiantes y docentes. Este significa disonancia, detenerse, parar, estar entre, generar dinámicas alternas y diferentes para reflexionar el trabajo social. Los integrantes de Interferencias han variado con el tiempo, algunos se mantienen hasta la actualidad, se han vinculado nuevas personas y otros por motivos diversos han abandonado el proceso. A todos los que han formado y forman parte de esta experiencia, un especial agradecimiento, por su participación y valiosos aportes.

${ }^{3}$ Las formas de trabajo del semillero desde el 2015, corresponden a un proceso de estudio reflexivo y vivencial sobre textos relacionados con la intervención e investigación del trabajo social en clave decolonial e intercultural. Los encuentros tienen una periodicidad quincenal, en espacios de la universidad. Semestralmente se realiza una reunión con las universidades que forman parte de la red en alguna de sus sedes. A partir del trabajo de varios semestres, se logran consolidar ideas generales de una investigación macro, que cada universidad apropia y construye según sus intereses y especificidades. En la actualidad, el semillero cuenta con un proyecto de investigación inscrito y avalado por la Universidad del Quindío, en saberes y resistencias de comunidades diversas.
} 
Factores para la formación en investigación del trabajo social: aportes desde la sistematización del semillero Interferencias...

estudiantes y docentes del semillero interferencias? En coherencia, el objetivo: comprender los factores del proceso de formación en investigación en trabajo social, que han contribuido al fortalecimiento de capacidades y actitudes investigativas de estudiantes y docentes del colectivo interferencias, entre el segundo semestre de 2015 y el primer semestre del 2017.

Luego de ubicar el interés de la sistematización, al retomar algunas de las fases propuestas por Jara (2012) para el diseño metodológico: se elaboró un plan de sistematización, desde el cual se delimitaron tiempos, fuentes de información, actividades e instrumentos ${ }^{4}$-entrevistas y observación participante-. Seguidamente, para la reconstrucción histórica, se realizó un proceso descriptivo de lo vivido, ordenando la experiencia cronológicamente y haciendo énfasis en las acciones y procesos desarrollados. Este ejercicio se acompañó de registros fotográficos, actas, relatorías, dibujos y demás documentos construidos en cada etapa.

Por último, se realiza el análisis e interpretación crítica, desde la cual se reflexiona sobre la experiencia, se reconocen las lecciones aprendidas, los cambios y nuevos sentidos construidos a partir de la misma.

Todo este proceso se abordó desde un enfoque cualitativo y comprensivo, con el fin de reconocer de manera intersubjetiva, los sentidos y significados construidos colectivamente por estudiantes y docentes del grupo, frente al proceso vivido. De allí que fuera necesario potenciar el protagonismo de los actores que formaron parte de la experiencia, ya que su perspectiva fue clave para la interpretación. En este sentido, la comunicación se convirtió en vehículo, para construir relaciones que permitieron avanzar en la comprensión, desde una perspectiva no jerárquica.

Teniendo en cuenta lo anterior, para esta sistematización, resultaron relevantes los aportes teóricos del construccionismo social (Gergen, 1996) en tanto enfatiza en lo relacional para la construcción de conocimiento y de la realidad. Invita a recuperar las relaciones colaborativas, la asociatividad y reciprocidad para asumir la intervención e investigación y construir colectivamente nuevas narraciones más inspiradoras. Esta mirada apreciativa motiva a asumir los procesos, los sujetos y las relaciones desde la potencia y la generatividad (Gergen, 1996).

Y la opción decolonial e intercultural (Gómez, 2015) porque permite cuestionar al profesional consumidor de conocimientos de otras disciplinas, para apostarle a la construcción de

\footnotetext{
${ }^{4}$ Los actores que aportaron en la reconstrucción de la experiencia fueron seis estudiantes y una docente que formaron parte del colectivo desde sus inicios y seguían participando a la fecha de la investigación. Como fuentes de información se rescataron actas de trabajo, fotografías, diarios de campo, dibujos, escritos y testimonios. Para avanzar en su rastreo y localización, se construyeron matrices que permitieran inventariar el registro de las experiencias; en estas, cada integrante del colectivo describía el tipo de registro con el que contaba, las reflexiones y aprendizajes de allí derivados. También se diseñó para la recolección de información, un plan de entrevistas que giraba en torno a los diferentes momentos vividos en la experiencia y las lecciones aprendidas.
} 
conocimientos que emergen de la experiencia y la praxis profesional. Este como elemento clave, invita a una lectura de la realidad desde otras epistemes, que hablen de lo propio, de lo local, que lleven a la visibilización de saberes negados históricamente, para que sean reconocidos y valorados.

\section{Resultados}

Las categorías de análisis que emergieron del proceso interpretativo fueron:

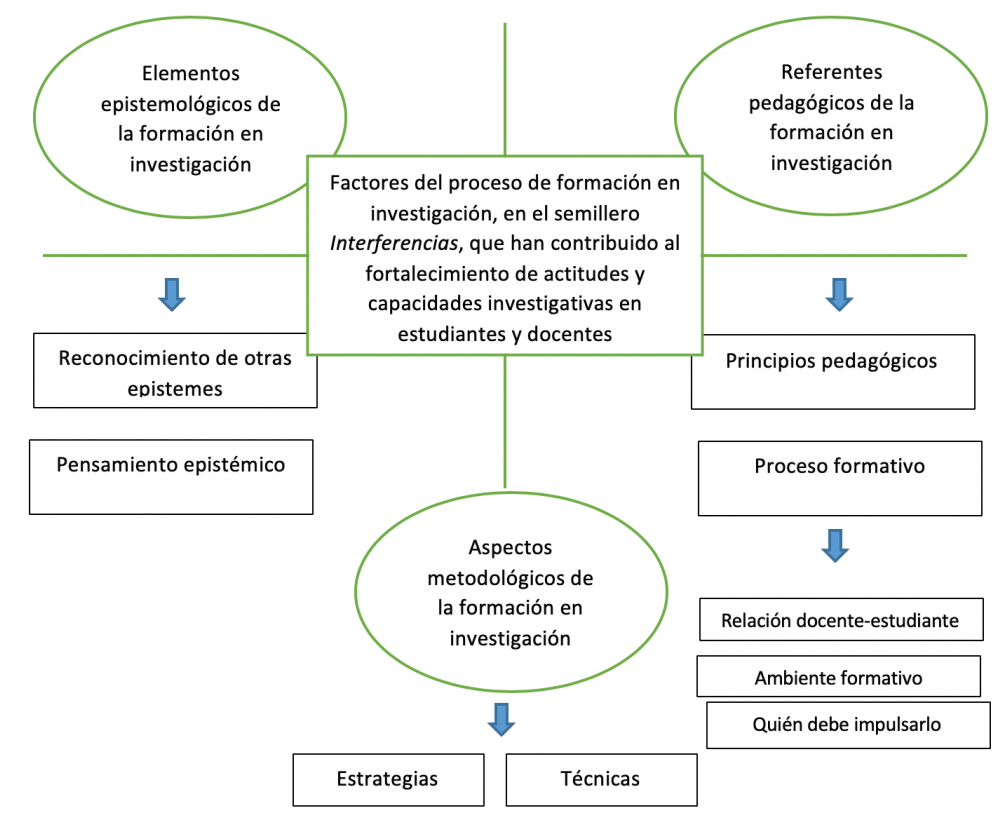

Ilustración 1. Categorías de análisis.

Fuente: elaboración propia.

\section{Elementos epistemológicos de la formación en investigación}

Como lo afirma Cifuentes (2013), resulta necesario asumir al trabajo social como profesión que aporta a la producción de conocimiento a partir de la investigación y hace énfasis en enfoques epistemológicos plurales, que superen la explicación causal de los fenómenos y la descripción de los mismos, dando apertura a ejercicios que permitan avanzar hacia la comprensión y transformación de procesos sociales complejos; lo anterior, por los alcances, y variedad de contextos y actores con los que la investigación se lleva a cabo desde la profesión. 
Factores para la formación en investigación del trabajo social: aportes desde la sistematización del semillero Interferencias...

A continuación, las categorías de análisis recuperadas, sobre los elementos epistemológicos que caracterizaron la formación en investigación para esta experiencia.

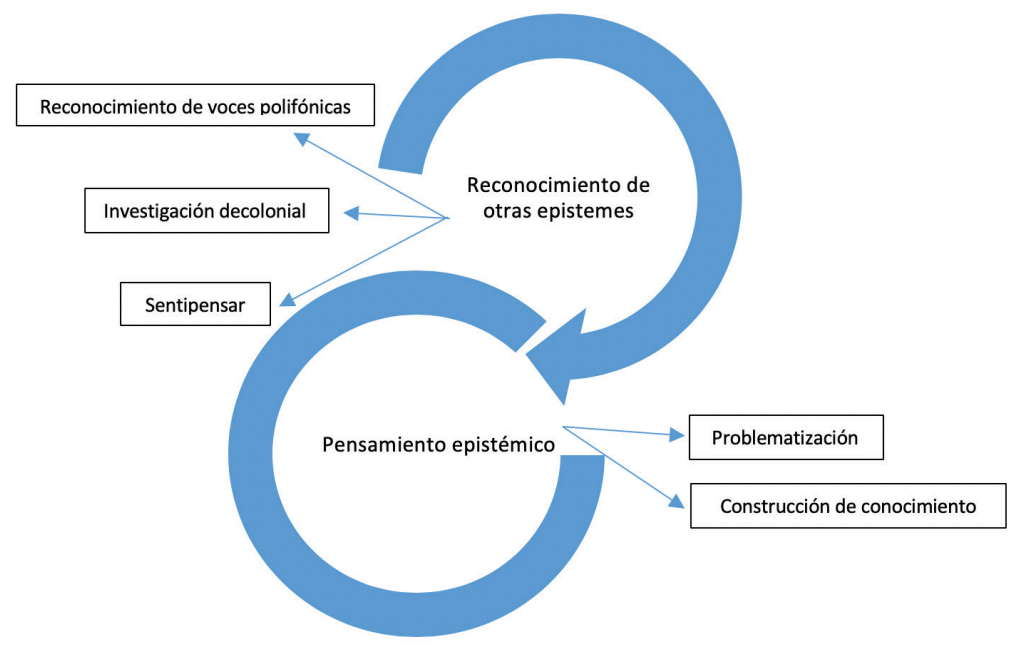

Ilustración 2. Construcción categorial: elementos epistemológicos de la formación en investigación.

Fuente: elaboración propia.

\section{Reconocimiento de otras epistemes}

\section{- Voces polifónicas}

El conocimiento científico como conjunto de hechos, evidencias y teorías que han sido validadas y obtenidas desde un método riguroso y único, cuenta con una aceptación incuestionada y es considerado como la única forma posible y legítimamente aceptada para producir conocimiento sobre la realidad. Al respecto, Mosquera (2005) citando a Walsh, afirma que:

(...) forma parte de las geopolíticas del conocimiento, la cual, a su vez, es una estrategia medular del proyecto de modernidad (...) Como conocimiento que se cree universal, oculta, invisibiliza y silencia otras epistemes. También oculta, invisibiliza y silencia los sujetos que producen ese otro conocimiento. (p. 266)

Es por este motivo que se hace un llamado a reconocer nuevos posicionamientos epistemológicos, para que no se desconozcan los sujetos (mujeres, indígenas, afros) y el conocimiento que construyen (Mosquera, 2005). 
Estos saberes locales generalmente son desconocidos y subvalorados, ya que el conocimiento científico aceptado y legítimo ha sido construido e importado de otros contextos con características sociales, económicas, culturales y políticas diferentes a las vividas en el sur.

En la actualidad, desde los discursos de la multiculturalidad y la puesta en marcha de los enfoques diferenciales, se ha tratado de abordar tal situación, sin embargo, este interés se queda en un ejercicio funcional y no trasciende hacia un reconocimiento, emparejamiento, y termina siendo una propuesta de adaptación de estas comunidades diversas al sistema dominante, (Walsh, 2009) o como lo propone Cusicanqui (Centro de Estudos Sociais, Laboratório Associado Universidade de Coimbra, 2013) un emblema fetiche, desde el cual pareciera que se está cumpliendo con las nuevas discusiones y exigencias, pero en realidad se va en contra vía de la experiencia.

Es por esto que resulta impostergable reconocer voces polifónicas, volver a nuestros propios contextos, para recuperar los conocimientos que de allí emergen, que se construyen desde las experiencias y que no son reconocidos por la ciencia formal. Igualmente es necesario transitar hacia la descolonización del saber, con el interés de evidenciar que existen otras formas de conocer, diferentes a las impuestas por la ciencia hegemónica, y otras formas de ser, de ver y de hacer. Al respecto los siguientes relatos.“...El discurso de occidente es el más escuchado, es la lengua más aceptada...” (Estudiante María Fernanda, comunicación personal, 2017).“...Solo nos importa escuchar discursos totalizantes y decir lo que dicen ellos, quedar como espejos de la cultura, no hacer parte de ella para crearla sino copiarla, pues es más fácil...” (Estudiante María Fernanda, comunicación personal, 2017).

Teniendo como base lo anterior, un elemento clave a rescatar del proceso vivido con el colectivo, es el hacer una lectura y reconocimiento para la práctica profesional desde otras epistemes, buscando asumir otros lentes (paradigmas) para comprender la realidad y tener en cuenta aquellos que hablen de lo propio, lo local, como sucede con las discusiones que ofrecen las epistemologías del sur.

Todo lo anterior, si bien invita a un ejercicio de recuperar los discursos propios, los saberes que se construyen en América Latina, no excluye el conocimiento de occidente, queda claro para los integrantes de Interferencias la necesidad de analizar el contexto de cada teoría, como lo propone Zemelman (2005) recuperar la lógica de construcción del autor, como construyó el problema y cómo lo teoriza, lo anterior se puede reflejar en el siguiente relato:

(...) las discusiones realizadas nos dejan claro que desde la perspectiva decolonial, no se invita a invalidar la teoría, o a rechazarla porque viene de otros contextos. De lo que se trata es formar un espíritu 
Factores para la formación en investigación del trabajo social: aportes desde la sistematización del semillero Interferencias...

autónomo, que logre posicionar su discurso, interpelar desde el propio contexto, debatir y argumentar. (Docente Ana María, comunicación personal, 2017)

La contextualización, análisis y argumentación aquí referidas, se asumen como capacidades para la investigación, las cuáles fueron movilizadas en los participantes del colectivo, desde el reconocimiento de diversos marcos epistemológicos.

\section{- Investigación decolonial}

Como se ha planteado, la investigación social tiene un compromiso ético y político con la vida, se trata de generar comprensiones que permitan abrir caminos hacia la construcción de mundos más justos y equitativos, donde se privilegie la garantía de derechos y dignidad humana. En las acciones lideradas por el grupo, la investigación ha ocupado un lugar principal como posibilidad para visibilizar, para dar voz a los que no se les escucha. Se trata de un ejercicio que no es lineal, ascensionista, pero sí comprometido, reflexivo, correlacional, en el que el sujeto que investiga se transforma, es impactado por "los otros", de los que también aprende. De aquí que sea necesario la superación de jerarquías, la cosificación de la población y por ende la descolonización de la investigación, ya que de alguna manera ha perpetuado prácticas hegemónicas y ha influido la matriz colonial.

Desde los aportes de Walsh (2008), la investigación es:

Una actividad que se lleva a cabo dentro de una serie de condiciones sociales y políticas, como también éticas y epistémicas. Por eso mismo y tal vez más que cualquier otro oficio académico, la investigación está arraigada a la problemática de la geopolítica del conocimiento y a la colonialidad: del saber, del ser y del poder, pero también a la colonialidad de la madre naturaleza o vida. (p. 2)

Es por esto que desde la investigación en clave decolonial, se construye conocimiento colectivamente, se reconocen saberes otros, se asume una perspectiva pedagógica en la que todos enseñan y todos aprenden.

Acorde con lo anterior, lo relacional cobra relevancia, con el objetivo de avanzar hacia un diálogo intercultural que trascienda la colonización del conocimiento desde la investigación, la cual conlleva a perpetuar discursos y teorías aceptadas universalmente, desconociendo los conocimientos y saberes producidos en otros contextos (Gómez, 2015). 
La opción decolonial invita a cuestionar la forma en la que se establece relación con el conocimiento y el papel que ocupa la ética en los procesos investigativos. Desde aquí, Gómez (2015) propone incorporar la pluralidad metodológica y establecer relaciones horizontales entre investigadores y actores sociales.

Estos aspectos fueron aprendizajes significativos para estudiantes:

\begin{abstract}
Llevaba poco tiempo con interferencias cuando fuimos al encuentro con el cabildo. Llegué con un mar de preguntas pensando en todo lo que ellos nos aportarían para la investigación, y no fue hasta un tiempo después que comprendí el gran error que había cometido, yendo a extraer conocimiento como si fueran objeto, sin reconocerlos (...) ellos de forma indirecta me enseñaron a apreciar y a escuchar lejos de lo que quisiera escuchar. (Estudiante Nicolás, comunicación personal, 2017)
\end{abstract}

La investigación social, entendida decolonialmente, permitió al grupo asombrarse frente a los asuntos referidos en este relato, en tanto ofreció elementos para problematizar como el poder permea el saber, en este caso, en cuanto al rol, metodologías, técnicas que asumen los investigadores, desde las cuales se instrumentaliza al otro y se privilegian los intereses individuales de quien investiga. Asumir nuevas formas de relacionamiento en los procesos investigativos, es el camino para aportar a la construcción de conocimiento plural en el que se cuente con la participación activa de diversos actores sociales.

\title{
- Sentipensar
}

Uno de los principales aspectos que ha marcado a integrantes del grupo es el sentipensar, encontrar un espacio en su proceso de formación académica, en el cual no se privilegie la separación de la razón con la emoción, resultó motivante para estudiantes y docentes que formaron parte del proceso.

Pasar por el cuerpo todo aquello que se estudia, analizarlo a la luz de los propios contextos y la experiencia vital, le otorga un sentido diferente a la problematización de las teorías propias de la formación disciplinar e investigativa. El sentipensar ofrece la posibilidad de asumir al sujeto en su totalidad, sin fragmentarlo, lo motiva a comprometerse con la construcción de otros mundos posibles y por tanto a cuestionar su realidad, a indignarse frente a ella.

Para Guerrero (2010), recuperar la afectividad negada en la academia por la hegemonía de la razón, es una práctica de resistencia de-colonial que permite "reintegrar la dimensión de totalidad de la condición humana, pues nuestra humanidad, descansa tanto en dimensiones de afectividad como de razón” (p. 89). Corazonar las prácticas pedagógicas y académicas 
Factores para la formación en investigación del trabajo social: aportes desde la sistematización del semillero Interferencias...

potenciará el reconocimiento de la alteridad, que estudiantes y docentes reconozcan la diferencia como posibilidad y no como amenaza.

Desde el semillero, en línea con el corazonar, se buscó que cada uno de los integrantes fuera libre de exponer sus ideas - sobre los textos estudiados- de la forma como más se emocionara y vinculara con ellas, buscando generar mayor reflexividad y apropiación. Fue gratificante encontrar cómo la música, dibujo, teatro y escritura abrirían las puertas al debate académico, sentido y pensado.

Al respecto Freire (1997a) plantea: "Para mí, es imposible conocer despreciando la intuición, los sentimientos, los sueños, los deseos. Es mi cuerpo entero el que socialmente conoce. No puedo en nombre de la exactitud y del rigor, negar mi cuerpo, mis emociones, mis pensamientos" (p. 128). El reconocimiento de estas ideas ofreció al grupo la motivación para continuar trabajando semestralmente a pesar de las dificultades de tiempo y la sobrecarga de funciones a las que se exponen estudiantes y docentes; asimismo, les permitió un mayor acercamiento y disfrute para con la investigación, reconocer un lado más humano de la ciencia y la academia. Es por esto que una de las principales reflexiones que emergen de esta experiencia con respecto a la formación investigativa es la necesidad de posibilitar ejercicios sentipensantes desde el escenario académico, lo cual incluye ejercicios desde el aula y fuera de ella:

Es necesario reconocer que, en la dinámica educativa actual, lo que
decimos no es de nosotros, hemos perdido nuestra identidad, no
escribimos desde el corazón, desde el sentir, tenemos mucho temor,
escribimos desde lo que dicen los autores, perdemos nuestra capacidad
de creatividad, de asombro. (Docente Ana María, comunicación
personal, 2017)

Esta idea permite problematizar la educación para recuperar capacidades menguadas a causa de la herencia de enfoque tradicional que privilegia la memoria sobre la reflexión, la certeza sobre la duda, la disciplina y el rigor metódico, sobre el aventurarse a la creación. Es por planteamientos como los anteriores que resulta posible considerar desde la experiencia vivida entre estudiantes y docentes, la importancia de abordar los procesos educativos desde marcos epistemológicos diversos (críticos, construccionistas, decoloniales) que dan paso a la problematización, creatividad y argumentación como actitudes propias de la investigación, a ser potenciadas en la formación profesional de los trabajadores sociales para su desempeño profesional. 


\section{Pensamiento epistémico}

Las principales características del pensar epistémico son la reflexión, la pregunta, el distanciarse de los constructos teóricos y aproximarse a la construcción de categorías que a diferencia de los conceptos tienen muchos contenidos (Zemelman, 2005).

Estos planteamientos han iluminado el trabajo en el grupo, según los relatos de sus participantes, ya que hacen reconocimiento explícito a la problematización, al sujeto situado y la construcción de conocimiento como asuntos que, de manera permanente, orientaron sus acciones.

\section{- Problematización}

Al referirse a la problematización de las ideas y los textos estudiados, se hace referencia al análisis contextuado, reflexivo, que parte de las experiencias vitales de quien la está agenciando. La pregunta juega un papel fundamental en este proceso y, desde la experiencia en el colectivo, se hizo constantemente la invitación a la duda, a la conversación con los autores más que a la repetición de sus teorías, buscando avanzar de la memorización a ejercicios de comprensión de las lógicas constructoras de los teóricos (Zemelman, 2005).

La problematización "significa estar dispuestos a zambullirse en el agua y comenzar a ver que hay más allá de la superficie, que hay debajo de la punta del iceberg” (Zemelman, 2005, p. 73), trascender de lo aparente y lo observable. Esta intención desde un principio, formó parte de los intereses del colectivo: promover espacios que propiciaran ver con otros ojos. Logramos escucharnos y dejar claro que nos interesaba construir un espacio para la reflexión, la problematización y el cuestionamiento.

Sin embargo, esta capacidad no resulta sencilla ya que lo acostumbrado es resguardarse en la teoría sin tener una posición argumentada frente a la misma. Para problematizar es necesario abrirse a las posibilidades, perder las certezas, aventurarse a lo incierto. Se trata de "descolocar" (Zemelman, 2005) los lugares que han ocupado tradicionalmente en el marco de una educación tradicional, docentes y estudiantes, unos validan y transmiten conocimientos, otros reciben y aprenden.

En el grupo, inicialmente hubo ciertas dificultades con respecto a la anterior idea, cuando se abrían los espacios para poner en práctica un pensamiento autónomo y problematizado, los ejercicios propuestos no lograban trascender a ello, pues siempre estaba presente el temor por fallar, por exponer ideas propias a un grupo conformado por docentes y compañeros. Otro de los panoramas evidenció que no había mucho que discutir, porque la forma en la que se hacen las lecturas de teorías, "se quedan en el procesamiento de contenido" (Zemelman, 2005, p. 75) y desde allí queda poco por argumentar y reflexionar. 
Factores para la formación en investigación del trabajo social: aportes desde la sistematización del semillero Interferencias...

\title{
- Construcción de conocimiento
}

Las concepciones antes expuestas, relacionadas con el conocimiento experto que poseen unos y la necesidad de validación de otros, son construcciones que han sido afianzadas desde los procesos formativos:

\begin{abstract}
Para este encuentro estábamos muy ansiosos, esperando que alguien nos aprobara o desaprobara lo que veníamos haciendo. Que nos dijera qué está bien y está mal (...) Todo lo contrario, ocurrió: reconocimos las voces polifónicas, las experiencias y ritmos particulares, cada quien se llevó las reflexiones que quiso, aprendizajes para seguir trabajando. (Docente Ana María, comunicación personal, 2017)
\end{abstract}

El temor y la incertidumbre que genera exponerse ante los demás, es posiblemente causa que limita hacer construcciones propias. Es común encontrar estudiantes y profesionales que se amparan en la teoría de forma textual, buscando una respuesta en ella para las complejas realidades que encuentran desde su intervención profesional.

Schön (1992) explica lo anterior como producto de una racionalidad técnica, de corte positivista, en la que es asumida la práctica como espacio para aplicación de conocimientos adquiridos en la academia. Es por esto que propone la necesidad de formar profesionales reflexivos que se posicionen de manera diferente frente a la práctica, al asumirla con creatividad y desde la planeación ${ }^{5}$, analítica de las situaciones que se le presentan.

Las relaciones pedagógicas juegan un papel fundamental en la formación de profesionales reflexivos, que se atrevan a aportar a la construcción de conocimiento desde sus prácticas, pues en el proceso educativo es cuando el docente puede motivar hacia la duda y la problematización. Sin embargo, lo que sucede usualmente es la construcción de una relación poco pareja, en la que estudiantes demandan respuestas exactas e inequívocas a sus profesores. Dudar es un proceso que para muchos implica insatisfacción, desasosiego, temor, razón por la cual se privilegian las respuestas puntuales y certeras de quienes enseñan, porque son considerados como poseedores absolutos del conocimiento.

Resulta pertinente romper con las lógicas tradicionales de relacionamiento, para construir nuevas interacciones en las que, quienes forman parte del acto educativo, sean asumidos como sujetos de conocimiento y a su vez de desconocimiento, de esta manera, todos serán participantes activos y constructores de reflexiones analizadas, en un ambiente respetuoso,

\footnotetext{
${ }^{5}$ Para el autor, el acto de planear, metafóricamente se refiere a la posibilidad de volar, y saber todo lo que ello implica, planear, aletear, esperar, enfrentar las tempestades (Schön, 1992).
} 
propicio para el aprendizaje colaborativo y para el rescate del sujeto en su propio discurso (Zemelman, 2005).

\begin{abstract}
He observado algo en la conversación que estamos llevando: algo que quiero denominar como prediscurso, discurso y posdiscurso. El prediscurso: está cargado de temores, excusas, incertidumbres, es casi como lanzarse al vacío con lo que quiero proponer. El discurso tiene que ver con mi mirada, mis reflexiones, mis apuestas frente a un tema, lo que para mí tiene significado. El posdiscurso tiene que ver con el silencio, el cual es aterrador, pero también como después de ese silencio se motiva la conversación desde lo que cada quien logró encontrar como clave... (Estudiante Nicolás, comunicación personal, 2017)
\end{abstract}

Lo anterior, en palabras de Zemelman (2005) implica reapropiar la teoría, enriquecerla, recuperando el acto de pensar, lo cual nuevamente enmarca la reflexión en las relaciones pedagógicas a partir de las cuales se genera un ambiente fecundo para la problematización y construcción del conocimiento. Al respecto, Aguirre (2014) plantea:

El conocimiento es una construcción humana y en consecuencia los sujetos son determinantes para su configuración, de allí que el profesor, adquiera un papel orientado al acompañamiento de los estudiantes, con el fin de generar preguntas, entendidas no en el sentido de validar conocimientos, sino en el camino de ampliar la mirada. (p. 27)

La responsabilidad del docente "es enseñar como conocer, enseñar a pensar correctamente" (Freire, 1997a, p. 135) y no enfocarse en los contenidos y la repetición de estos, si bien es importante conocer las teorías, resulta fundamental apropiarlas y problematizarlas.

Es por esto que la responsabilidad del docente en la formación de actitudes investigativas desempeña un papel principal, su compromiso, rigor e interés por el proceso formativo de los estudiantes se refleja en el acompañamiento y motivación hacia la generación espacios propicios para el debate, la argumentación, y por ende el aprendizaje y construcción colectiva del conocimiento.

\title{
Referentes pedagógicos de la formación en investigación
}

La formación integral de los estudiantes es una intención central en los procesos de formación en investigación, de allí que sea necesario discutir sobre los asuntos de orden pedagógico que potencian tales procesos. Desde esta experiencia, se encontraron los siguientes aspectos: 


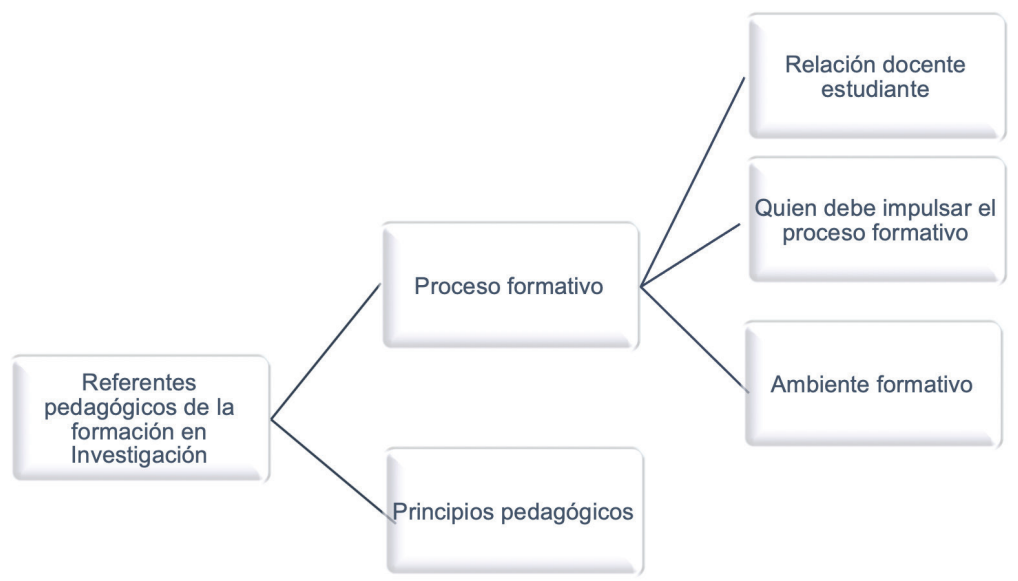

\section{Ilustración 3. Construcción categorial: referentes pedagógicos de la formación en investigación. \\ Fuente: elaboración propia.}

\section{Proceso formativo}

Tener conciencia de los principios que orientan los procesos formativos, la relación que se construye entre estudiantes y docentes además del ambiente y tipo de enseñanza que se privilegia, permitirán identificar el o los enfoques pedagógicos que se asumen al momento de formar en investigación y establecer los giros o énfasis que sean necesarios en cada caso.

\section{- Relación estudiantes y docentes}

Construir una relación cercana, caracterizada por el respeto, la confianza, el reconocimiento y la horizontalidad, es un proceso que demanda tiempo, dedicación y el aprovechamiento de espacios diferentes al aula de clase donde sea posible conocer al otro más allá de su rol de docente y estudiante. Desde esta experiencia, el reconocimiento, al igual que el fortalecimiento de las relaciones, fue un aspecto a resaltar: "La primera salida oficial (...) una experiencia muy agradable, dónde se plantearon objetivos, también se expresaron expectativas, se compartieron momentos muy lindos que fortalecieron el colectivo (...)” (Estudiante Jennifer, comunicación personal, 2017).

Establecer relaciones cercanas, no atemorizantes, en las que se privilegie el trabajo colaborativo, permiten trascender el autoritarismo y verticalidad propios a una propuesta pedagógica tradicional de la educación y motiva una actitud autónoma, reflexiva y crítica, en los procesos de formación investigativa. Anteriormente se había planteado la importancia de generar espacios para la potenciación del pensamiento epistémico, orientado por la pregunta 
y la problematización. Al referirse a las relaciones entre estudiantes y docentes, este aspecto vuelve a tomar fuerza, en tanto, a partir de esta dinámica se logran desestructurar los rangos y jerarquías entre profesor, estudiante, como el que posee saber y el que no, ya que todos se encuentran dispuestos y motivados a potenciar la reflexión y la argumentación no solo desde la teoría sino desde su experiencia particular.

Acorde a lo anterior, las pedagogías decoloniales realizan una crítica al poder que permea el saber científico, universal y legítimamente válido. Por tanto, se motiva a un reconocimiento de saberes otros y se motiva hacia una mayor porosidad entre el conocimiento que se gesta en la académica y el saber popular, al considerarlos igualmente importantes. Esto obliga a establecer de manera diferente las relaciones entre universidad y comunidad, en este caso, entre docentes y estudiantes, en tanto todos son asumidos como sujetos con saberes.

La relación pedagógica que acá se privilegia está caracterizada por el todos enseñan, todos aprenden, razón por la cual, las interacciones que se gestan en el proceso educativo aportan a quienes forman parte del mismo:

\begin{abstract}
Cada encuentro que tengo con el colectivo me deja con motivación, energía. Me sorprende la capacidad de reflexión y emoción de los estudiantes, de algunos especialmente frente a la vida, lo cotidiano, los textos revisados. Considero que me aportan, a veces siento que me muestran caminos que antes no había contemplado, me aclaran (...)

(Docente Ana María, comunicación personal, 2017)
\end{abstract}

Salir del lugar que tradicionalmente el docente ha ocupado en el proceso educativo y abrirse a la posibilidad de escuchar, promueve el establecimiento de relaciones más democráticas y horizontales, las cuales resultan claves para acompañar con compromiso y rigor el proceso de formación en investigación el cual implica también para el docente posibilidad de aprendizaje y apertura a nuevas experiencias.

\title{
- Quién debe impulsar el proceso formativo
}

Si bien los estudiantes forman parte fundamental del proceso formativo y son responsables de su aprendizaje en tanto son protagonistas de este, quien impulsa el proceso educativo tiene gran responsabilidad en la creación de ambientes y condiciones necesarias para que tal aprendizaje sea significativo. Al respecto, Freire (1997a) plantea: "El maestro tiene el deber de revivir, de renacer a cada momento de su práctica docente para que los contenidos que enseña sean algo vivo y no nociones petrificadas" (p. 121). 
Factores para la formación en investigación del trabajo social: aportes desde la sistematización del semillero Interferencias...

Por tanto, ir en coherencia con las nuevas formas y dinámicas de enseñanza, aprendizaje, estar al tanto de los debates disciplinares contemporáneos y hacer lecturas renovadas de los ejes formativos que aborda, es una responsabilidad que debe asumir el docente en el acompañamiento que realiza, en este caso, desde la formación para la investigación.

Tal acompañamiento, según Cifuentes (2014), parte de reconocer las capacidades de quien aprende, sus ritmos e intereses. Por tal motivo el docente requiere asumir una actitud de escucha que le permita comprender cada caso en su particularidad para motivar, orientar e inspirar el proceso de quienes están aprendiendo a investigar, investigando.

La escucha implica "que se emparejen los que hablan y los que escuchan, si no se hace, no hay diálogo, tampoco se acepta el escuchar, de aquí que sea importante concederle más importancia al otro, y menos al hablar" (Lenkersdof, 2008, p. 30); en esta dirección, existe disposición para aprender del otro y para comprenderle, situación que posiciona diferente al docente en el proceso educativo.

El llamado a la comprensión, persistencia, escucha y trabajo colectivo, al reconocimiento de los aportes que cada uno realiza, sus talentos y aspectos a fortalecer en cuanto a la investigación, son algunas de las responsabilidades que desde esta experiencia se lograron identificar para el docente que acompaña procesos de formación investigativa. En este caso, estas actitudes favorecieron un ambiente de confianza y compromiso, lo cual se vio reflejado posteriormente en la creatividad y apertura a la conversación.

La actitud del docente para la formación en investigación, debe estar orientada por un proceso de acompañamiento riguroso (Cifuentes, 2014), que contempla asuntos de orden metodológico, pero principalmente de reflexión crítica sobre la realidad y sobre las teorías. El docente que acompaña procesos de investigación en trabajo social debe mostrarse convencido de su importancia para la intervención profesional, además de esto, vivir la investigación como parte fundamental de su labor. Según Falla (2012): "Una de las estrategias pedagógicas más importantes que debe desarrollar, es la de incentivar a la comprensión de la investigación como un proyecto de vida y no como una tarea momentánea” (p. 17), como una opción para el desarrollo de prácticas fundamentadas y comprometidas con los horizontes y fines profesionales, la dignidad, derechos humanos, justicia social y construcción de paz.

\section{- Ambiente formativo}

El ambiente que se construye en un proceso formativo juega un papel importante en tanto puede posibilitar o dificultar el aprendizaje, la potenciación de capacidades y el aprovechamiento de las sesiones educativas. En el caso de la formación investigativa con estudiantes universitarios, 
resulta necesario propiciar un entorno que favorezca el cuestionamiento, la reflexión crítica de la realidad a investigar, el compromiso y la pasión por esta.

El reconocer la experiencia vital de cada integrante, sus talentos y habilidades, generó un ambiente cálido, de interés y motivación por las acciones a desarrollar, esto a su vez permitió avanzar en cada encuentro hacia la reflexión y problematización: "Como consecuencia de este ejercicio, surgen cartas, me di cuenta de la capacidad de reflexionar sobre los otros y sobre mí. Todos estos ejercicios, son mi trampolín y desfogue”. (Estudiante María Fernanda, comunicación personal, 2017)

Esto propició un mayor acercamiento y compromiso entre sus integrantes, quienes a su vez crearon una atmósfera propicia para la escucha, la emoción y la conversación, aspectos que impactan en el reconocimiento de la diferencia como potencia y no como amenaza: "El otro no tiene por qué ser como yo, debo escuchar para poder entender por qué” (Estudiante Sofía, comunicación personal, 2017).

La escucha es condición central de la conversación, esta implica ponerse en el lugar del otro, acercarse, emparejarse, para escuchar y ser escuchados, para transitar de las individualidades al nosotros (Lenkersdof, 2008), lugar desde el cual se manifiesta la reciprocidad, ya que se reconoce la importancia de lo que todos tienen para expresar así sea en condiciones de marcadas diferencias y desacuerdos.

Escuchar implica reconocer la existencia de sí mismo y el lugar que los otros ocupan en esa existencia, lo cual permite dejar de verlos como rivales y antagonistas para encontrar aquello que los acerca. El acercamiento favorece la construcción de relaciones abiertas al diálogo y a la conversación, tal como sucedió en la experiencia sistematizada.

\footnotetext{
El ejercicio de la escucha es lo más significativo de este semestre, ha sido transversal a los acontecimientos de mi vida en este momento, escuchar las voces que no son propias pero que generan ecos, para bien o para mal. Esto aporta a lo personal. (Estudiante Manuel, comunicación personal, 17 de mayo de 2017)
}

La conversación implica participación, dejar de lado el monólogo "o conversación en un solo sentido, como aquella en la que una o varias ideas monopolizan u ocupan todo el espacio" (Anderson, 2005, p. 65), para involucrar a los otros de manera activa y recíproca. Al respecto, Anderson (2005) plantea:

Las conversaciones generadoras implican un proceso activo, en el que ambas partes participan por igual, (...) en un ir y venir, en el que las 
Factores para la formación en investigación del trabajo social: aportes desde la sistematización del semillero Interferencias...

personas hablan unas con otras, no unas a otras, creando significados juntas. El psicólogo John Shotter (1993) se refiere a este tipo de conversación, como "una conversación en la que la gente siente que pertenece”, una comunidad local en la que se crean significados, y las personas sienten que pueden contribuir en algo y ser escuchadas. (p. 64)

El conversar en los procesos formativos, propicia ambientes que favorecen la reflexión y desde allí una construcción conjunta de nuevas miradas y comprensiones frente a los temas trabajados. Desde los procesos para la formación en investigación, es necesario enfocarse en construir un clima que propicie la emoción, reflexión y compromiso con la investigación, con los principios y fines del trabajo social y "con la producción y apropiación social del conocimiento, como fundamento de la práctica profesional” (Cifuentes, 2013, p. 172).

\section{Principios pedagógicos}

Asumidos como las condiciones necesarias para orientar el proceso educativo, generalmente se construyen en coherencia con las metas formativas, los lineamientos curriculares y permiten direccionar los ambientes educativos y prácticas docentes con el objetivo de apuntar al mejoramiento, calidad e integralidad en la formación.

Los principios pedagógicos que orientaron el proceso vivido en el grupo fueron: el trabajo colaborativo, autonomía del sujeto, reflexividad y fortalecimiento de redes interinstitucionales.

El trabajo colaborativo se enmarcó en lo relacional, en resolver, abordar situaciones en colectivo, se privilegió el trabajo por grupos y desde allí la construcción conjunta de nuevos significados frente a los temas, problemáticas o realidad abordada. Como se ha planteado a lo largo del documento, el reconocimiento del otro, de sus aportes y reflexiones, han sido fundamentales para el trabajo, lo cual enmarca el proceso en un enfoque apreciativo que rescata lo generativo, viable y posible.

¿Quién soy yo sin el otro? Este proceso que iniciamos desde primer semestre me ha permitido conocer, comprender y construir con el otro, a mis compañeros y a mí mismo. Así también fue posible reconocer el crecimiento de todos desarrollando diversas aptitudes. (Estudiante Manuel, comunicación personal, 2017)

Las diversas aptitudes referidas invitan a reconocer que, si bien lo relacional ha sido fundamental en el trabajo realizado, también lo es el reconocimiento del sujeto como protagonista, cada quien, de manera autónoma, establece su propio estilo, ritmo de reflexión y aprendizaje. Si bien existen unas metas colectivas, cada uno es constructor activo de su proceso y decide hasta dónde llega con el mismo. Es por este motivo que el grupo se muestra diverso, 
heterogéneo y las capacidades que se han fortalecido en cuanto a la investigación varían de un sujeto a otro, cada quien se ha encargado de darle el énfasis que considera según sus intereses y potencialidades. "Es querer escribir, es querer entender, pero también desentender, me lleva a desdibujar, a reescribir mi paso por la vida” (Estudiante María Fernanda, comunicación personal, 2017).

Asumir la autonomía como principio pedagógico del grupo potenció, a su vez, la libertad y creatividad en sus integrantes. Si bien en el relato anterior se hace referencia explícita a la escritura, se pudieron reconocer en este proceso otras formas de trabajar los objetivos propuestos, entre ellas la fotografía, el dibujo, la música, todas ellas influyeron en el ambiente de trabajo y en la motivación para continuar en el mismo.

En línea con lo anterior, la reflexividad motivó la capacidad de asombro y el análisis detenido de las situaciones a estudiar ya que, para el grupo, siempre fue importante hacer lecturas diversas, profundas que llevaran a dudar, a desaprender, a dejar de lado las certezas para darle paso a la incertidumbre. "Reflexioné sobre mi auto-indignación, me critiqué a mí mismo, conociéndome como nunca lo había hecho” (Estudiante Cristian, comunicación personal, 2017).

Con respecto a las redes interinstitucionales, desde el trabajo en el grupo se privilegió el encuentro con semilleros y grupos de investigación de otras universidades, a través de sesiones de trabajo en las que se debatían y profundizaban temas relacionados con la investigación en curso: el trabajo social desde la perspectiva decolonial e intercultural.

Establecer conexiones con otros amplió el panorama de reflexión, se reconocieron diferentes ritmos, estrategias y formas de trabajo que inspiraron al grupo y lo motivaron a continuar cada vez con mayor compromiso.

\section{Aspectos metodológicos de la formación en investigación}

La metodología en los procesos educativos permite trazar la ruta y orientar las acciones de docentes y estudiantes. Esta se encuentra en concordancia con los lineamientos pedagógicos y se desglosa en métodos, estrategias y técnicas que aportan al cumplimiento de los objetivos de formación. En esta experiencia, se reconocen como aspectos centrales de lo metodológico, los siguientes: 


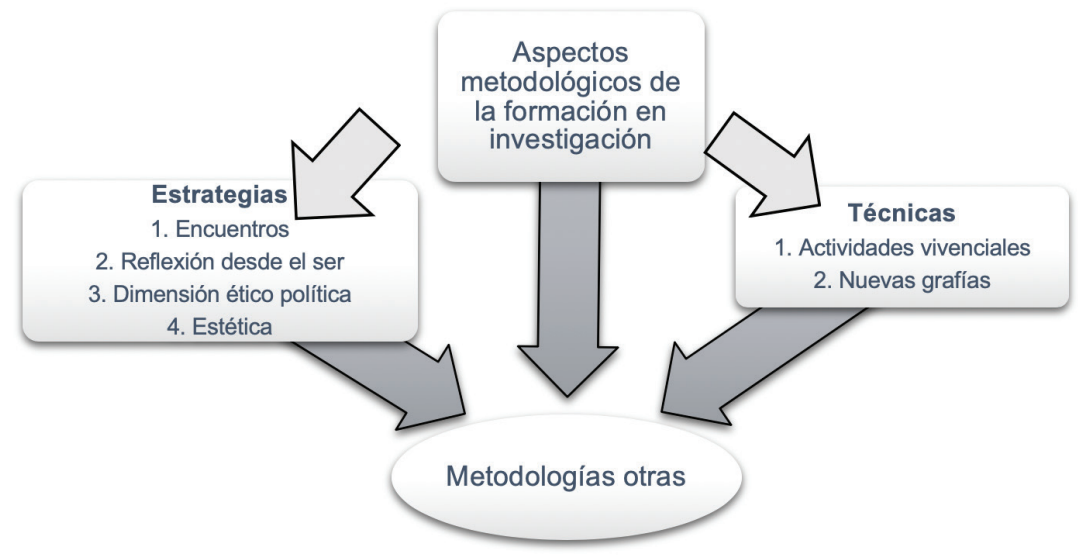

Ilustración 4. Construcción categorial: aspectos metodológicos de la formación en investigación.

Fuente: elaboración propia.

\section{Estrategias y técnicas}

El grupo trabajó de diferentes formas para cumplir con sus objetivos. Principalmente se hizo a partir de encuentros locales y departamentales. Desde el trabajo local se estableció una dinámica de reunión quincenal para avanzar en la socialización y debate de los compromisos adquiridos. A nivel departamental, se acordaron encuentros semestrales con los semilleros y grupos de investigación de las universidades que forman parte de la red. "Con la Universidad de Caldas, trabajamos en la investigación, mostramos nuevas actividades que se habían realizado, tuvimos un compartir de experiencias, también se planearon nuevos objetivos para socializarlos en el siguiente encuentro" (Estudiante Jennifer, comunicación personal, 2017).

En estos espacios se compartían avances, fortalezas y dificultades vividas por cada colectivo, lo cual permitió tejer relaciones de apoyo, acompañamiento para enfrentar situaciones, además de ampliar las reflexiones y conocimiento frente a los temas abordados desde la experiencia compartida.

Los encuentros permitieron el contacto cara a cara, implicaron presencialidad y responsabilidad con el cumplimiento de compromisos. A partir de estos espacios de reflexión, se construyeron conversaciones y diálogos de saberes para avanzar en el proceso de aprender a investigar, investigando.

Otra de las estrategias que se logró identificar como característica del trabajo por parte del grupo es la reflexión desde el ser, contextualizar lo estudiado, a la situación del país y 
a la experiencia vital, amplió las posibilidades de reflexión, asombro e indignación, frente a diversas situaciones de la realidad social. Asimismo, motivó cambios autónomos y personales.

\begin{abstract}
Con el foto-sentir, vi el significado de otras cosas (...) Dudas, miedos. La foto de la malla significó obstáculos de la vida. Con el ejercicio del tejido, estaba triste y encontré que todo tiene sentido, el hilo de la vida. En el encuentro entre universidades, por lo que logramos hacer, reafirmé la importancia de encontrar amigos. Desde la actividad de la escucha (...) me di cuenta de cosas, de escucharme a mí misma y a los otros. (Estudiante María Fernanda, comunicación personal, 17 mayo de 2017)
\end{abstract}

Lo anterior, como aprendizaje para los procesos de formación en investigación es relevante, ya que permite comprender como el sujeto que investiga también se transforma y es impactado por los otros.

Así mismo, en este último relato se logran apreciar algunas de las técnicas a partir de las cuáles se materializaron los encuentros locales e interuniversitarios: a. El reconocimiento de nuevas grafías: cuentos, poemas, dibujos, fotografías que invitan a "sobrepasar el peso colonial de que lo escrito es lo que existe, pues nos abrimos a la creatividad que otorga aprender nuevos lenguajes que han sido menospreciados como medio comunicativo" (Gómez, 2015, p. 9). b. Actividades vivenciales: círculos de palabras, encuentros con la naturaleza, actividades de tejido y barro, como metodologías otras, que se alejan de la dinámica tradicional académica y posibilitan el fortalecimiento de capacidades para el asombro, escucha, creación, observación, diálogo y argumentación, además del disfrute y el compartir. Freire (1997b) plantea lo siguiente: “(...) las dimensiones en las que se es posible aprender críticamente (...) implican o exigen la presencia de educadores y de educandos creadores, instigadores, inquietos, rigurosamente curiosos, humildes y persistentes" (p. 28).

Desde la experiencia del grupo, tal proceso de aprendizaje crítico también fue posible a partir de la estética como una estrategia clave para expresar ideas, liberar sentidos y formas diversas de representar el mundo. En este sentido, se rescata la idea propuesta por Vázquez y Barrera (2016), quienes proponen trascender la mirada de la estética colonial, que regula las subjetividades y lo bello desde una perspectiva eurocéntrica hacia una aesthesis, que lleve a visibilizar otras formas de ver, comprender y habitar el mundo, las cuales han sido ocultas por la modernidad colonial.

Estas otras formas de asumir lo metodológico, desde la experiencia en el grupo, influyeron en el fortalecimiento de destrezas y actitudes hacia la investigación y, a su vez, motivaron la movilización y acción colectiva para visibilizar la complejidad de la realidad social y 
Factores para la formación en investigación del trabajo social: aportes desde la sistematización del semillero Interferencias...

pronunciarse frente a ella. Ante las inconformidades de los estudiantes, pero con la esperanza de un cambio, hablamos del plebiscito por la paz. Se pegaron carteles generando conciencia y un poco de reconocimiento por las decisiones que se tomarán, afectando a los ya afectados. (Estudiante Jennifer, comunicación personal, 2017)

En esta línea, la dimensión ético-política como estrategia para fortalecer la capacidad de acción frente a lo que concierne al sujeto de manera individual, pero que a su vez compromete a los otros, aporta a la construcción de ciudadanos comprometidos con su país y con la transformación de situaciones que vulneren los derechos y atenten contra la dignidad humana y la justicia social.

\section{Discusión}

La formación en investigación es necesaria para los estudiantes de trabajo social quienes, desde su ejercicio profesional, deben desplegar con solvencia actitudes investigativas (Grassi, 2011) que les permitan desempeñarse rigurosamente en la intervención. Desde esta experiencia de sistematización, los factores que inciden positivamente en la formación investigativa de estudiantes y docentes se enmarcan en aspectos de orden epistemológico, pedagógico y metodológico. Esto significa que tal proceso debe ser estructurado de manera consciente, por los docentes que se interesen en acompañar investigaciones de estudiantes, de allí que sea necesario cuestionar: los principios pedagógicos, relaciones y rol docente, ambientes formativos, técnicas, estrategias y horizontes epistemológicos desde los cuales se trabaja y materializa el proceso.

Por tanto, reconstruir esta experiencia fue útil: a). Para el Semillero, pues permitió a sus integrantes reflexionar sobre su proceso; reconocer aquello necesario de potenciar; b). Para la academia, ya que posibilita abrir el debate sobre escenarios emergentes, alternativos de educación, en donde más que los contenidos memorizados prima la reflexión, el sentir y el reconocimiento de saberes diversos. c). Para los estudiantes y profesionales, porque tendrán referencia de otras formas de intervención/ investigación, en las que se privilegie lo relacional y se asuma "al otro" como protagonista válido desde sus intereses, saberes y conocimientos.

Con respecto a las potencias del estudio, se rescata: la suficiencia de memorias (actas, fotografías, escritos, dibujos) con las que se contaron para reconstruir rigurosamente la experiencia, acceso directo a partir de entrevistas, observaciones y demás técnicas, a la voz de los actores, sistematización liderada por un integrante del Semillero que vivió toda la experiencia del grupo.

En cuanto a las limitaciones: la dificultad de participación por parte de todos los integrantes del colectivo para la fase de interpretación y análisis crítico de la experiencia. 
Principalmente el tiempo de los estudiantes, desde sus ocupaciones académicas limitaron el encuentro para avanzar en reflexiones propias a la sistematización. De allí que metodológicamente una recomendación para futuras experiencias sea apostar a diferentes alternativas para el análisis y trabajo colaborativo que no impliquen necesariamente la presencialidad de los actores.

Así mismo, es recomendable continuar con estudios que permitan ampliar el debate sobre la formación en investigación, desde esta experiencia, los factores epistemológicos, pedagógicos y metodológicos rescatados se encuentran en línea con los aportes de la opción decolonial e intercultural y el construccionismo social, contrario a los trabajos rastreados y publicados en el tema, los cuales se enmarcan en otro tipo de modelos pedagógicos que si bien ofrecen valiosos aportes, se enmarcan en propuestas convencionales de la educación. De aquí la necesidad de innovar, aventurarse a lo desconocido y apostarle a pedagogías y metodologías otras para acercarse a la problematización del conocimiento, apasionarse en el proceso y relacionarse de manera diferente con los estudiantes.

\section{Conclusión}

Potenciar capacidades y actitudes investigativas en estudiantes y docentes requiere compromiso, disciplina y el despliegue de factores epistemológicos, pedagógicos y metodológicos incluyentes, diversos, coherentes con los nuevos tiempos y contextos en los que habitamos, desde los cuales se asuman estudiantes y docentes como sujetos de conocimiento, se invite a la problematización, reflexión y cuestionamiento. Se trata de reconocer que cada uno tiene algo para decir, de allí la importancia de potenciar el diálogo, la argumentación y el debate, privilegiando formas diversas para hacerlo. Desde este marco, resulta importante motivar a los estudiantes para que expongan y argumenten sus ideas, en eventos y diferentes escenarios académicos, esto les permite generar seguridad y apropiarse de sus discursos, para que no repitan de memoria lo enseñado desde los textos académicos y, por el contrario, conversen con las teorías, las interpelen y contextualicen.

Teniendo en cuenta lo anterior, es necesario re-inventarse, trascender las miradas totalizadoras, eurocéntricas y coloniales sobre la vida, la academia y la investigación, dejándose permear por otras comprensiones que invitan a la creatividad y a asumir los procesos de manera cíclica, no lineal y etapista, desde los cuales lo relacional, generativo y potenciador, resulten aspectos clave para construir prácticas profesionales reflexivas y emancipadoras.

La formación para la investigación debe ser continua y procesual, no debe responder solamente a requisitos de orden institucional, ya que demanda otros tiempos, otras formas de trabajo que no necesariamente van amarradas a las directrices de los programas académicos. Esto implica, como se vivió en la presente sistematización, motivar la vinculación de estudiantes a semilleros 
Factores para la formación en investigación del trabajo social: aportes desde la sistematización del semillero Interferencias...

y grupos de estudio desde los primeros semestres, con el objetivo de ir construyendo una dinámica de trabajo y reflexión diferente a la generada en el aula de clase, desde la cual los estudiantes no se acostumbren a trabajar por el incentivo de la nota sino por el compromiso de su formación personal y profesional. Institucionalizar estos espacios, hacerlos visibles en el contexto académico y de allí establecer redes de trabajo intra e interuniversitarias, son opciones para darle mayor impulso y reconocimiento a los procesos.

Finalmente, enfrentar la incertidumbre que generan propuestas formativas enmarcadas en discusiones alternativas y apostarles desde el trabajo constante, con los actores involucrados en ellas, evitando que sean permeadas por la lógica positivista, cientificista y tradicional, desde la cual se desconocen e invalidan discursos como el sentipensar y la potencia de los saberes locales, es un desafío para quienes están interesados en otras formas de ver la realidad, la práctica profesional y la investigación. Resistir, re-existir, como apuesta de vida y alternativa de construcción de otros mundos, más justos, equitativos, diversos.

\section{Referencias}

Aguirre, J.S. (2014). La investigación formativa en Trabajo Social: una propuesta para fortalecer actitudes investigativas. Bogotá, Colombia: Consejo Nacional para la Educación en Trabajo Social-CONETS.

Anderson, H. (2005). Un enfoque posmoderno de la terapia. La música polifónica y la terapia desde adentro. En G. Limon. (Ed.), Terapias posmodernas, aportaciones construccionistas (pp. 59-67). Ciudad de México, México: Editorial Pax.

Centro de Estudos Sociais, Laboratório Associado Universidade de Coimbra (productor). (2013). Conversas do mundo IV. (video). Recuperado de http://www.youtube.com/.

Cifuentes, R.M. (2014). Acompañar la formación investigativa, investigando. IV encuentro Latinoamericano de Metodología de las Ciencias Sociales (ELMeCs). Encuentro realizado en la Universidad Nacional de la Plata, la Plata, Argentina.

Cifuentes, M.R. (2013). Formación en Trabajo Social e Investigación: una relación insoslayable de cara al siglo XXI. Revista de Trabajo Social, (15), 165-182.

Falla, R.U. (2012). La investigación, eje transversal en la formación en trabajo social en Colombia. Espacio Regional, 1 (9),13-27.

Freire, P. (1997a). Educación en la ciudad. Ciudad de México, México: SXXI Editores.

Freire, P. (1997b). Pedagogía de la autonomía. Saberes necesarios para la práctica educativa. Ciudad de México, México: Siglo XXI Editores. 
Gergen, K. (1996). Realidades y relaciones, aproximaciones a la construcción social. Barcelona, España: Paidós.

Gómez, H.E. (2015). Investigación decolonial desde trabajo social. En N. Burgos (presidencia), La formación profesional el Trabajo Social: Avances y tensiones en el contexto de América Latina y el Caribe. Conferencia realizada en el XXI Seminario Latinoamericano de Escuelas de Trabajo Social, Mazatlán, México.

Grassi, E. (2011) La producción en investigación social y la actitud investigativa en el trabajo social. Revista Debate Público. Reflexión de Trabajo Social, 1 (1), 128-139. Recuperado de http: / / trabajosocial.sociales.uba.ar/wp-content/uploads/sites/13/2016/03/16_ grassi.pdf.

Guerrero, A. P. (2010). Corazonar el sentido de las epistemologías dominantes desde las sabidurías insurgentes, para construir sentidos otros de existencia. Bogotá, Colombia. Calle 14 revista de investigación en el campo del arte, 4 (5), 80-94.

Jara, O.H. (2012). La sistematización de experiencias práctica y teoría para otros mundos posibles. San José, Costa Rica: Editores Alforja y Concejo de Educación de Adultos de América Latina (CEAAL).

Lenkersdof, C. (2008). Aprender a escuchar. Enseñanzas Maya Tojolabales. México D.F., México: Plaza y Valdés editores.

Mosquera, R.C. (2005). Pluralismos epistemológicos: hacia la valorización teórica de los saberes de acción. Una reflexión desde la intervención social de la población afrocolombiana desplazada. Palimpsestvs. Revista de la Facultad de Ciencias Humanas de la Universidad Nacional de Colombia, 5, 262-276.

Schön, D. (1992). La formación de profesionales reflexivos. Barcelona, España: Editorial Paidós.

Vázquez, R. y Barrera, M. (2016). Aesthesis decolonial y los tiempos relacionales Entrevista a Rolando Vázquez. Calle 14, Revista de Investigación en el Campo del Arte, 11(18), 76-93. DOI: https://doi.org/10.14483/udistrital.jour.c14.2016.1.a06.

Walsh, C. (2008). (De) Colonialidad e investigación. Ética, práctica y saber. Ponencia presentada en el I Encuentro Internacional y V Nacional de investigación, Universidad Libre, Bogotá, Colombia.

Walsh. C. (2009). Temas de interculturalidad crítica desde Abya yala. Chiapas, México: Universidad de la Tierra.

Zemelman, H. (2005). Voluntad de conocer: el sujeto y su pensamiento en el paradigma crítico. Ciudad de México, México: Anthropos. 